EXTENDED REPORT

\title{
Vitrectomy with short term postoperative tamponade using perfluorocarbon liquid for giant retinal tears
}

\author{
M Sirimaharaj, C Balachandran, W C Chan, A P Hunyor, A A Chang, J Gregory-Roberts, \\ A B Hunyor, T J Playfair
}

See end of article for authors' affiliations

Correspondence to: Maytinee Sirimaharaj, Vitreoretinal Unit, Mettapracharak Hospital (Watraikhing), 52 Moo 2, Raikhing District, Sampran, Nakornpathom 73210, Thailand; maytinees@yahoo.com

Accepted for publication 1 March 2005
Br J Ophthalmol 2005;89:1176-1179. doi: 10.1136/bjo.2004.065409 Aim: To determine the efficacy and safety of perfluorocarbon liquid
tamponade in patients with retinal detachment from giant retinal tears. Method: A retrospective consecutive case series of patients with retinal detachment from giant retinal tears who underwent vitrectomy using perfluorocarbon liquid as a short term postoperative internal tamponade. The perfluorocarbon liquid was removed 5-14 days (mean 7.5 days) later and replaced by gas or silicone oil. Scleral buckling was performed in some cases with proliferative vitreoretinopathy. The crystalline lens was removed if there was interference with the surgical view or if it was subluxated. The success rate of retinal reattachment, visual outcome, and postoperative complications were assessed.

Results: A total of 62 eyes of 61 patients with a follow up of 8-69 months (mean 24.5 months) were included. All retinas were attached intraoperatively. 14 eyes $(22.6 \%)$ developed re-detachment and additional operations were performed in 13 eyes. At final visit, 58 eyes $(93.5 \%)$ had retinas that remained attached with visual acuity $6 / 12$ or better in 27 eyes (46.5\%). The visual acuity improved in 34 eyes (54.8\%) with 28 eyes (45.2\%) improving at least two Snellen lines, it was unchanged in 20 eyes (32.3\%), and was worse in eight eyes (12.9\%). Three patients developed glaucoma that was controlled medically. There was no retained perfluorocarbon liquid in any eyes.

Conclusion: Perfluorocarbon liquid appears safe and effective to use as a short term postoperative tamponade in management of retinal detachment from giant retinal tears.
G iant retinal tears (GRT) are retinal breaks extending circumferentially for 90 degrees or more. $^{12}$ The surgical approach for this condition has always been a challenge for vitreoretinal surgeons, as these patients have a high risk of proliferative vitreoretinopathy (PVR) formation (40-50\%). ${ }^{3}$ Many approaches ${ }^{2-8}$ to repositoning and fixating the inverted retinal flap, reattaching the retina, and reducing the risk of redetachment have been reported with varying success rates.

The use of perfluorocarbon liquids (PFCLs) demonstrated by Chang et $a l^{4}$ to unfold and flatten the inverted retina provides several advantages. Most reported series ${ }^{3-7}$ used the PFCLs only as an intraoperative tool, and demonstrated an increase in success rate. However, the rate of recurrent retinal detachment was still high, a major problem being slippage of the retinal flap. In this study, we analysed our results with long term follow up of treating retinal detachment associated with GRT using PFCLs as a short term postoperative tamponade.

\section{PATIENTS AND METHODS}

The charts of 65 consecutive patients who underwent surgery for retinal detachment from GRT between l February 1997 and 31 January 2004 in the Vitreoretinal Unit, Sydney Eye Hospital, were reviewed retrospectively. Follow up data were sought from referring local ophthalmologists where they had been involved in the postoperative management of the patients. Four patients were excluded; two were overseas and another two were lost to follow up.

\section{Surgical technique}

A standard three port pars plana vitrectomy was performed. The vitreous was cut and aspirated meticulously and endodiathermy was used to mark the edge of the tear(s)
The infusion bottle height was lowered and perfluoro-noctane $\left(\mathrm{PFO}, \mathrm{C}_{8} \mathrm{~F}_{18}\right)$ was then injected slowly to produce a single bubble completely filling the vitreous cavity using a 20 gauge blunt or silicone tipped cannula, placed above the optic disc to unroll the folded retina. Endophotocoagulation and/or indirect laser was then applied around the tears and to the basal retina for $360^{\circ}$ in all eyes. Cryotherapy was applied in 35 eyes, only to the two ends of the GRT. Scleral buckling was performed in the eyes with preoperative PVR and no previous buckle. Lensectomy was performed if cataract obscured the surgical view or the lens was subluxated.

The patients were positioned according to the location of their GRT-usually lying on the ipsilateral side, on alternate sides for very extensive GRT, or upright in the cases of predominantly inferior GRT, for 5-14 days (mean 7.5 days, median 7 days). Then, vitrectomy with PFO/fluid (balanced salt solution, BSS) exchange was performed, followed by fluid/air and air/gas or silicone oil exchange. Sulphur hexafluoride $\left(\mathrm{SF}_{6}\right) 20 \%$, perfluoropropane $\left(\mathrm{C}_{3} \mathrm{~F}_{8}\right) 12 \%-14 \%$, or silicone oil (1300 or 5700 centistokes) were used for exchange with PFO.

Retinal reattachment, recurrent retinal detachment, and additional procedures, final visual outcome, and complications were assessed.

\section{RESULTS}

Sixty two eyes of 61 patients were included; 51 patients were male and 10 were female, with ages ranging from 4 years to 63 years (mean 44.2 years). Ten eyes were highly myopic (range -6 dioptres to -20 dioptres), three had a history of penetrating trauma, and 16 had blunt trauma. One patient

Abbreviations: BSS, balanced salt solution; GRT, giant retinal tears; PFCLs, perfluorocarbon liquids; PFO, perfluoro-n-octane; PVR, proliferative vitreoretinopathy; $R P E$, retinal pigment epithelium 


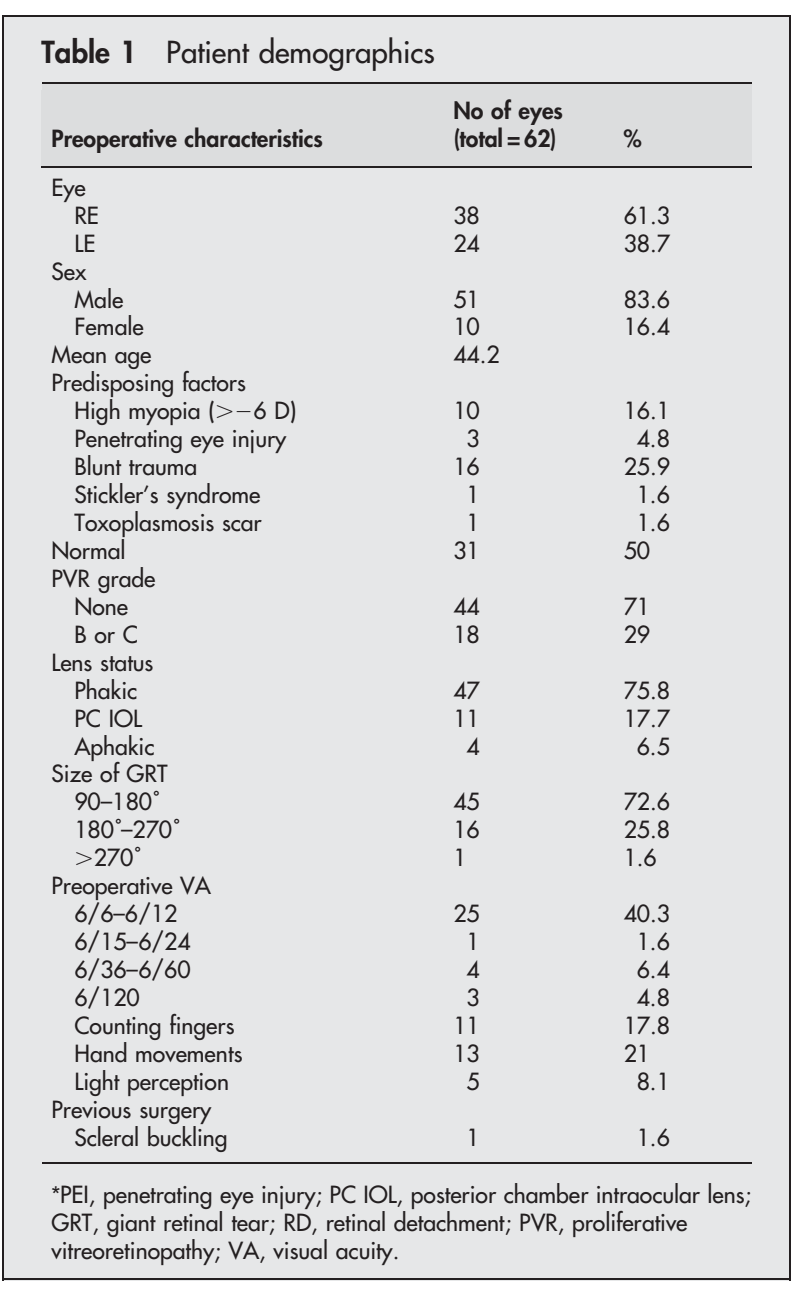

had Stickler's syndrome and one had a GRT extending from the edge of an inactive toxoplasmosis scar. Forty seven eyes were phakic, 11 were pseudophakic with posterior chamber implants, and four eyes were aphakic (three as a results of penetrating injury and one following surgery for congenital cataract).

The patient demographics are shown in table 1 . The preoperative visual acuity was $6 / 12$ or better in 25 eyes, 6/15 to $6 / 24$ in one eye, $6 / 36$ to $6 / 60$ in four eyes, $6 / 120$ in three eyes, counting fingers in 11 eyes, hand movements in 13 eyes, and light perception in five eyes. The tears were less than $180^{\circ}$ in 45 eyes, $180^{\circ}$ to $270^{\circ}$ in 16 eyes, and more than $270^{\circ}$ in one eye. PVR was grade B or C (Retina Society Classification) ${ }^{9}$ in 18 patients. Concurrent scleral buckling was performed in 17 eyes; one eye already had failed conventional scleral buckling 3 days previously. Lensectomy was performed in two eyes because of the presence of cataract, one of which was subluxated. $\mathrm{SF}_{6}, 20 \%$, was used in 42 eyes, $\mathrm{C}_{3} \mathrm{~F}_{8}, 12 \%-14 \%$, in

Table 2 Summary of operative procedures

\begin{tabular}{lcr}
\hline Operative procedures & $\begin{array}{r}\text { No of eyes } \\
\text { (total=62) }\end{array}$ & $\%$ \\
\hline Scleral buckling & 17 & 27.4 \\
Lensectomy or phacoemulsification & 5 & 8.1 \\
PFO exchange in $2^{\circ}$ surgery to & 4 & \\
Balanced salt solution & 42 & 6.5 \\
$20 \% \mathrm{SF}_{6}$ & 11 & 67.7 \\
$12 \%-14 \% \mathrm{C}_{3} \mathrm{~F}_{8}$ & 5 & 17.7 \\
Silicone oil (1300 or $5700 \mathrm{cS}$ ) & 5.1 \\
\hline
\end{tabular}

11 eyes, and five eyes had silicone oil as secondary internal tamponade. Four eyes were left with BSS. All retinas were attached intraoperatively. Three eyes developed progressive cataract between these two operations, so the cataracts were removed at the time of PFO removal. The summary of operative procedures is shown in table 2 .

Minimum follow up was 8 months, with a mean period of 24.5 months (median 18 months; range 8-69 months). At 3 months postoperatively, 50 of the $62(80.6 \%)$ eyes had completely reattached retinas, 12 eyes had recurrent retinal detachment at a mean period of 26.7 days (range 5-42 days) after removal of PFO. Eight of these had increasing PVR, three developed new retinal tears, and one had reopening of the GRT. Another two eyes developed re-detachment more than 3 months postoperatively; one eye had progressive PVR, the other had history of blunt trauma with pre-existing glaucoma and developed a re-detachment after removal of silicone oil for uncontrolled high intraocular pressureultimately this eye was enucleated.

A total of 13 eyes with re-detachment underwent a further vitrectomy. Only one of these had combined scleral buckling and vitrectomy for re-detachment (six eyes already had scleral buckling from previous surgery). Two eyes had concurrent lensectomy and three eyes had phacoemulsification with posterior chamber implants. Silicone oil was used in 10 eyes, one eye had $20 \% \mathrm{SF}_{6}$, and two eyes had $14 \% \mathrm{C}_{3} \mathrm{~F}_{8}$. Ten eyes were reattached, three eyes were still detached and considered inoperable. At final visit, the anatomic success rate was 58 of 62 eyes (93.5\%).

Of the 47 phakic eyes preoperatively, a total of 10 cataracts were removed at the same time of surgery for retinal detachment because of interference with visualisation of the posterior segment. One patient developed high intraocular pressure secondary to forward displacement of the crystalline lens from $\mathrm{C}_{3} \mathrm{~F}_{8}$, so lensectomy with posterior chamber implant and removal of $\mathrm{C}_{3} \mathrm{~F}_{8}$ was performed and the retina remained attached. Cataracts developed in 29 of 36 eyes $(80.5 \%)$; 20 eyes $(55.5 \%)$ had cataract surgery with a mean time of 13.7 months (range 4-30 months) post-retinal surgery. Glaucoma developed in three eyes $(4.8 \%)$ following retinal reattachment surgery; two eyes were highly myopic ( -15 dioptres and -20 dioptres $)$ and one eye had a history of blunt trauma and had been filled with silicone oil. The intraocular pressure was controlled medically in these three eyes. There was no residual PFO visible in any eyes.

At the last follow up, the visual acuity was $6 / 12$ or better in 27 eyes, 6/15 to 6/24 in nine eyes, 6/36 to 6/60 in 11 eyes, $6 / 120$ in four eyes, counting fingers in four eyes, hand movements in five eyes, light perception in one eye, and no light perception in one eye. There was improvement in visual acuity in 34 eyes $(54.8 \%)$ with 28 eyes $(45.2 \%)$ showing an improvement of at least two Snellen lines. Visual acuity was unchanged in 20 eyes (32.3\%) and deteriorated in eight eyes $(12.9 \%)$, of which two had cataracts, one developed a macular hole, and five had surgery for re-detached retinas which resulted in reattachment in three and persistent

Table 3 Outcome at last postoperative visit

\begin{tabular}{lll}
\hline Outcome measure & $\begin{array}{l}\text { No of eyes } \\
\text { (total =62) }\end{array}$ & $\%$ \\
\hline Attached retinas & 58 & 93.5 \\
Improved VA ( $\geqslant 2$ Snellen lines) & $34(28)$ & $54.8(45.2)$ \\
No change & 20 & 32.3 \\
Worse VA & 8 & 12.9 \\
VA $\geqslant 6 / 120$ & 51 & 82.3 \\
VA $\geqslant 6 / 12$ (of attached retina) & 27 & 46.5 \\
\hline & &
\end{tabular}




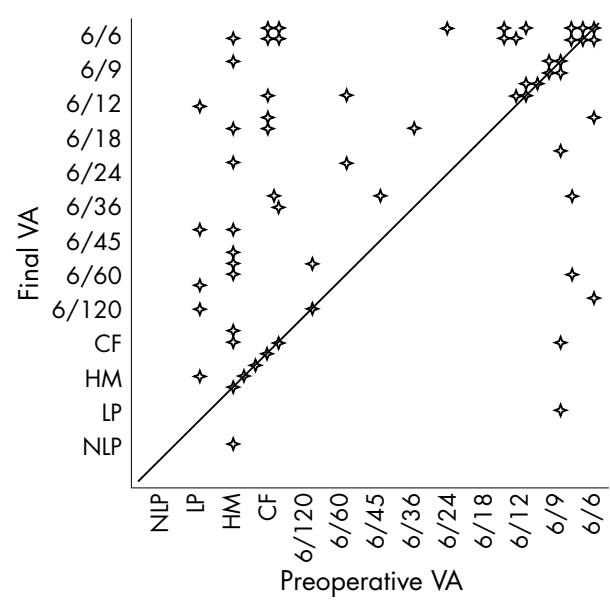

Figure 1 Scatter plot comparing preoperative and final visual acuity (VA). NLP, no light perception; LP, light perception; HM, hand movements; $\mathrm{CF}$, counting fingers.

detachment in two. At the last visit, $46.5 \%$ of the reattached eyes had a visual acuity of $6 / 12$ or better. The summary of final outcomes is shown in table 3. The comparison of preoperative and final visual acuity is shown in figure 1.

\section{DISCUSSION}

Our series of 62 patients is comparable to previously published series in terms of patient demographics (with the usual male predominance), and predisposing factors-most commonly trauma and high myopia, with $50 \%$ of cases being idiopathic. $^{23^{10-12}}$ Several studies ${ }^{3-6}{ }^{13-21}$ conclude that PFCLs are safe and useful as an intraoperative adjunct in complicated retinal detachment with severe PVR, diabetic retinopathy, and GRT. Chang et al ${ }^{4}$ reported the final reattachment in 16 of 17 eyes (94\%) with GRT. However, nine eyes (52.94\%) needed further treatment for re-detachment and some patients had posterior slippage of the retinal tears during air/fluid exchange, which was corrected after turning the patients to prone position. The report from the Vitreon Collaborative Study Group ${ }^{17}$ demonstrated a re-detachment rate of $49.4 \%$ in 162 eyes with GRT, which needed additional surgery and a final success rate of reattachment in $90.7 \%$. Perfluoroperhydrophenanthrene was used as an extended postoperative tamponade for 3-1034 days (mean 87.2 days) in 16 eyes $(9.9 \%)$. The Perfluoron Study Group ${ }^{18}$ used PFO as an intraoperative tamponade in 212 eyes with GRT and reported a final outcome of $76 \%$ retinal reattachment, and $30 \%$ reoperation for re-detachment.

Perfluorodecalin, perfluoroperhydrophenanthrene, and PFO have been reported to be well tolerated in eyes for at least 5 days. $^{22}{ }^{23}$ Chang et al ${ }^{24}$ reported occasional macrophages containing oil-like vacuoles on the inner surface of the inferior retina without ultrastructural changes in the outer plexiform layer and photoreceptor outer segments in rabbit eyes containing PFO for up to 1 week. Focal areas of narrowing of the outer plexiform layer and ultrastructural distortions of photoreceptor outer segments in the inferior retina were noted after 2 weeks. ${ }^{24}$ These changes may represent a mechanical rather than toxic effect, as similar changes have been reported in the superior retina in silicone filled eyes. ${ }^{24}$ As PFCLs are biologically inert and appear to be well tolerated for longer term tamponade, we used PFO as an intraoperative tool and as a postoperative tamponade. We elected to leave PFO in situ for 5-14 days, as this provided time for sufficient chorioretinal adhesion and allowed scheduling of a logistically convenient time to remove the PFO.
In our series, we achieved final anatomic success in 58 of 62 eyes $(93.5 \%)$ which was similar to previous reports by Chang et al $^{4}(94 \%)$ and the Vitreon Collaborative Study Group $^{17}(90.7 \%)$; however, the rate of re-detachment in this study was lower $\left(22.6 \%\right.$ compared to $52.9 \%{ }^{4}$ and $\left.49.4 \%{ }^{17}\right)$. Similar results have been reported in other studies which used PFCLs as postoperative tamponade. Blinder $e t a^{25}$ used perfluoroperhydrophenanthrene in 16 eyes with complicated retinal detachment including four eyes with GRT. The perfluoroperhydrophenanthrene was left for 5 days to 4 weeks with four of 16 eyes (25\%) needing further operation for re-detachment and all retinas were attached at the last visit. Bottoni et $a l^{26}$ reported leaving perfluorodecalin for 5 days postoperatively in the management of GRT. In their series, the retina reattached in nine of 11 eyes $(82 \%)$ and two $(18 \%)$ had additional surgery for re-detachment. All retinas were attached at final follow up.

It has been suggested that the lower rate of re-detachment when using PFCLs as postoperative tamponade may occur as a result of the extended apposition of the retinal tear to the underlying retinal pigment epithelium (RPE) resulting in more effective chorioretinal adhesion by retinopexy, ${ }^{46}$ so reducing the risk of reopening or posterior slippage of retinal tears. The incidence of inferior PVR is also lower because of lack of pooling of RPE cells, chemoattractants, and serum components over the inferior retina. ${ }^{25}{ }^{26}$ Postoperative positioning is also significantly easier for patients with PFO as opposed to gas or silicone oil, especially with inferior GRT.

At the last visit, $46.5 \%$ of the attached retinas in our study had visual acuity of $6 / 12$ or better and 51 of 62 eyes $(82.3 \%$ ) had visual acuity $6 / 120$ or better which was not significantly different from the results of Chang et al ${ }^{4}(88.2 \%)$.

There was no serious adverse effect of using PFO in this study. The rate of cataract progression in our patients was $80.5 \%$. This finding was not different from previous reports of cataract progression $(70 \%-80 \%)$ after simple vitrectomy for idiopathic epiretinal membrane ${ }^{27}$ and from Brazitikos et al ${ }^{15}$ (87\%), who used PFCLs as an intraoperative tool for retinal reattachment.

In conclusion, our study demonstrated that PFO appears effective and safe when used as a short term postoperative tamponade in cases of retinal detachment from GRT. This technique reduces the rate of re-detachment and also the need for additional operations. The obvious disadvantage of this technique is the requirement of secondary surgery for removal of PFO.

\section{Authors' affiliations}

M Sirimaharaj, C Balachandran, W C Chan, A P Hunyor, A A Chang, J G Roberts, A B Hunyor, T J Playfair, Vitreoretinal Unit, Sydney Hospital and Sydney Eye Hospital, Sydney, Australia

\section{REFERENCES}

1 Schepens CL, Dobbie JG, McMeel JW. Retinal detachments with giant retinal breaks: preliminary report. Trans Am Acad Ophthalmol Otolaryngol 1962;66:471-8.

2 Schiff W, Chang S, Reppucci V, et al. Surgical management of giant retinal tears. In: Guyer DR, eds. Retina-vitreous-macula. Vol 2, eds. Pennsylvania: WB Saunders, 1999:1338-49.

3 Ghosh YK, Banerjee S, Savant V, et al. Surgical treatment and outcome of patients with giant retinal tears. Eye 2004;18:996-1000.

4 Chang S, Lincoff H, Zimmerman NJ, et al. Giant retinal tears. Surgical techniques and results using perfluorocarbon liquid. Arch Ophthalmol 1989; 107:761-6.

5 le D, Glaser BM, Sjaarda RN, et al. The use of perfluoro-octane in the management if giant retinal tears without proliferative vitreoretinopathy Retina 1994; 14:323-8.

6 Glaser BM, Carter JB, Kuppermann BD, et al. Perfluoro-octane in the treatment of giant retinal tears with proliferative vitreoretinopathy. Ophthalmology 1991;98:1613-21.

7 LoRusso FJ, Diaz-Rohena R, Lambert HM. Management of giant retinal tears. Sem Ophthalmol 1995;10:42-8. 
8 Peyman GA, Schulman JA, Sullivan B. Perfluorocarbon liquids in ophthalmology. Surv Ophthalmol 1995;39:375-95.

9 Machemer R, Aaberg TM, Freeman HM, et al. An updated classification of retinal detachment with proliferative vitreoretinopathy. Am J Ophthalmol 1991;112:159.

10 Aylward GW, Cooling RJ, Leaver PK. Trauma-induced retinal detachment associated with giant retinal tears. Retina 1993;13:136-41.

11 Chaudhry NA, Flynn Jr HW, Tabandeh H. Idiopathic giant retinal tears in identical twins. Am J Ophthalmol 1999;127:96-9.

12 Bourke RD, Cooling RJ. Perfluorocarbon heavy liquids. Aust N Z J Ophthalmol 1995;23:165-71.

13 Kreiger AE, Lewis $\mathrm{H}$. Management of giant retinal tears without scleral buckling. Ophthalmology 1992;99:491-7.

14 Comaratta MR, Chang S. Perfluorocarbon liquids in the management of complicated retinal detachments. Curr Opin Ophthalmol 1991;2:291-8.

15 Brazitikos PD, Androudi S, D'Amico D, et al. Perfluorocarbon liquid utilization in primary vitrectomy repair of retinal detachment with multiple breaks. Retina 2003;23:615-21.

16 Batman C, Cekic O. Vitrectomy with silicone oil or long-acting gas in eyes with giant retinal tears. Retina 1999;19:188-92.

17 Kates PJ, Wafapoor H, Peyman GA, Vitreon Collaborative Study Group, et al. The management of giant retinal tears using perfluoroperhydrophenanthrene. Ophthalmology 1997;104:1159-65.

18 Scott IU, Murray TG, Flynn HW, and the Perfluoron Study Group, et al. Outcomes and complications associated with giant retinal tear management using perfluoro-n-octane. Ophthalmology 2002;109:1828-33.
19 Imamura YI, Minami M, Ueki $M$, et al. Use of perfluorocarbon liquid during vitrectomy for severe proliferative diabetic retinopathy. Br J Ophthalmol 2003;87:563-6.

20 Shaikh S, Trese MT. Retinal reattachment facilitated by short-term perfluorocarbon liquid tamponade in a case of FEVR and rhegmatogenous retinal detachment. Retina 2002;22:674-6.

21 Millsap CM, Peyman GA, Mehta NJ, et al. Perfluoroperhydrophenanthrene (Vitreon) in the management of giant retinal tears: results of a collaborative study. Ophthalmic surg 1993;24:759-62.

22 Eckardt C, Nicolai U, Winter M. Experimental intraocular tolerance to liquid perfluorooctane and perfluoropolyether. Retina 1991;11:375-84.

23 Bottoni F, Sborgia M, Arpa P, et al. Perfluorocarbon liquids as postoperative short-term vitreous substitutes in complicated retinal detachment. Graefes Arch Clin Exp Ophthalmol 1993;231:619-28.

24 Chang S, Sparrow JR, Iwamoto T, et al. Experimental studies of tolerance to intravitreal perfluoro-n-octane liquid. Retina 1991;11:367-74.

25 Blinder KJ, Peyman GA, Desai UR, et al. Vitreon, a short-term vitreoretinal tamponade. Br J Ophthalmol 1992;76:525-8.

26 Bottoni F, Bailo G, Arpa P, et al. Management of giant retinal tears using perfluorodecalin as a postoperative short-term vitreoretinal tamponade: a long-term follow-up study. Ophthalmic Surg 1994;25:365-73.

27 Cherfan GM, Michels RG, de Bustros S, et al. Nuclear sclerotic cataract after vitrectomy for idiopathic epiretinal membranes causing macular pucker. Am J Ophthalmol 1991;111:434-8. 